\title{
Review on targeted drug delivery against intracellular pathogen
}

\begin{abstract}
Targeted drug delivery into the cell compartment that is the most vulnerable to effects of the corresponding drug is a challenging problem, and its successful solution can significantly increase the efficiency and reduce side effects of the delivered therapeutic agents. The current technologies for increasing of bioavailability of antibiotics by means of liposomes or nano particles. These carriers were preferentially developed because their composition is compatible with biological constituents. The in vivo behavior of both types of carriers and consequently their therapeutic potential, are determined by their route of administration. Conventional carrier strategies permit the mononuclear phagocyte system to be targeted by intravenous injection of antibiotics. Stealthy strategies avoid major uptake by these cells and extend the systemic presence of these carriers. The purpose of this review is to provide background information in drug deliveries targeting intracellular pathogens gathered from papers published over the last twenty years. It seems clear that such drug carriers (liposomes, nanoparticles) allow increased drug concentration at infected sites but reduce drug toxicity. Herein, I focus on the application of nanotechnology and liposomes to drug delivery and highlight several areas of opportunity where current and emerging nanotechnologies could enable entirely novel classes of therapeutics.
\end{abstract}

Keywords: targeted drug delivery, intracellular infection, liposome, nanoparticle
Volume 6 Issue 3 - 2018

\author{
Nigat Atbiaw, ${ }^{2}$ Endris Aman, ${ }^{2}$ Bereket \\ Dessalegn, ${ }^{2}$ Oumer Masrie, ${ }^{2}$ Demeke \\ Debalke, ${ }^{2}$ Gashaw Enbiyale,' Andnet Yirga, ${ }^{2}$ \\ Gebrehiwot Tekilu, ${ }^{2}$ Askale Abrhaley, ${ }^{2}$ \\ Fentahun Mitku² \\ 'Field Practitioner, College of Veterinary Medicine and science, \\ University of Gondar, Ethiopia \\ ${ }^{2}$ Candidate of Veterinary medicine, College of Veterinary \\ Medicine and science, University of Gondar, Ethiopia
}

Correspondence: Nigat Atbiaw, Veterinary medicine, College of Veterinary Medicine and science, University of Gondar, Ethiopia,Email nigatatibiyaw@gmail.com

Received: April 14, 2018 | Published: May 15, 2018

\section{Introduction}

Delivering therapeutic compound to the desirable site is a major problem in treatment of many diseases. Conventional utilization of drugs is characterized by poor biodistribution, limited effectiveness, undesirable side effects, and lack of selectivity. The distribution of an active molecule in the body is mainly a function of its physicochemical properties. An alternative approach to the classical delivery of antibacterial therapy consists in associating the drug to a submicroscopic carrier thereby hiding and protecting the molecule from degradation and delivering it to inaccessible target cells in a controlled manner. It defined as the 'magic bullet. ${ }^{1}$ Liposomes and nanoparticles are the main carriers developed for these logistic targeting strategies and are colloidal in nature, biodegradable and similar in behaviour to intracellular pathogens. The ability of circulating carriers to target these cells is highly dependent on tissue characteristics and on the carrier's properties. Immediately after injection, the foreign particles are subjected to opsonization by plasma proteins. In this way, 'classical or conventional carriers' are recognized by the mononuclear phagocytic system. ${ }^{2}$ Pathogenic microorganisms are sometimes able to survive and reproduce after they have been ingested by phagocytic cells, especially macrophages, which hinder the treatment of these types of infection. The intracellular locations of these microorganisms are protecting them from host defense mechanism and from the action of antibiotics, which may encounter difficulties in penetrating phagocytic cells. ${ }^{3}$ The main objectives of this review are to analyze the different non biological and biological delivery system used to improve the uptake of drugs by infected host cells.

\section{Major types of drug delivery against intracellular pathogen}

Microorganisms in infected tissues are protected by various biological structures around the infection foci. Indeed, the adhesion properties of bacteria are also expressed by secreting glycocalyx in pathological conditions providing increased protection and hence increased resistance to antibacterial agents. ${ }^{4}$ Despite the discovery of new antibiotics, the treatment of intracellular infections often fails completely to eradicate the pathogens. ${ }^{5}$ By loading antibiotics into the colloidal carriers, liposomes and nanoparticles, one can expect improved delivery to infected cells. ${ }^{6}$ Interest in liposomes is directed upon their vesicular structure limited by one or more outer protecting shell(s) consisting of lipids arranged in a bilayers configuration and upon their ability to interact with living cells in one of four ways: adsorption, endocytosis, lipid exchange and fusion. ${ }^{7}$ Currently there are two major types of delivery systems:-liposomes and nanoparticles.

\section{Liposomes}

Liposomes are currently in common use as universal drug carriers in the cosmetic and pharmaceutical industries. In healthcare there are antitumor anthracyclines such as doxorubicin and antifungal amphotericin B liposomal formulations available. ${ }^{8,9}$ The starting point of the discovery of liposomes was a wonderful artificial model for the biological membrane developed by Dervichian, Luzzati and Chapman. These phospholipid dispersions in water solutions were able to trap and release solutes, to which they were selectively permeable. ${ }^{10}$ The word 'liposome' is derived from the Greek: 'lipo' referring to their fatty constitution and 'soma' referring to their structure. ${ }^{11}$ Gregoriadis 
used the property of sequestration of solutes by liposomes to formulate the concept of the liposome drug-carrier. Usually, liposomes are classified into three categories on the basis of their size and lamellarity (number of bilayers): small unilamellar vesicles (SUVs) or oligolamellar (OLVs), large unilamellar vesicles (LUVs) and multilamellar vesicles (MLVs). Entrapment efficiency is limited by the volume of the liposomes and drug solubility. The active compound can be located either in the aqueous spaces, if it is water-soluble, or in the lipid membrane, if it is lipid soluble. Liposomes have been administered in a variety of ways but intravenous injection is the most practical route. The intracellular penetration of liposomes is effected through endocytosis when they should become localized in endosomes where degradation by phospholipases takes place. ${ }^{12,13}$ Lipid vesicles as drug carriers significantly influence on drug distribution and reduce toxic side effects during antibiotic therapy. ${ }^{14}$

Structure and properties of liposomes: Liposomes are spherical vesicles consisting of one or more phospholipid bilayers surrounding a water space. The diameter of the liposome varies from 0.02 to $10 \mathrm{~mm}$. Vesicle formulations are usually based on natural and synthetic phospholipids and cholesterol. ${ }^{15}$ The structure may also possess lipoproteins.

The physicochemical properties of liposomes can be modified by changing:

a. The types of lipids.

b. The composition and proportions of lipids in the liposomal formulation.

c. The size of the liposome.

d. The charge of the liposomal surface: positive, negative, or neutral $\mathrm{pH}$ sensitivity.

e. Temperature sensitivity.

f. The fluidity of the liposomal membrane: rigid and fluid liposomes. ${ }^{16}$

Regarding the variety of liposomal formulations, the vesicles are universal carriers for both hydrophilic and hydrophobic compounds. Hydrophilic elements are dissolved in the water space inside the vesicles. The most useful for this are LUVs (Large Unilamellar Vesicles) because the volume of encapsulated water is relatively high hydrophobic compounds are located in the lipid bi layer, and MLVs (Multi lamellar Vesicles) or SUVs (Small Unilamellar Vesicles) can be applied. Charged drugs can be associated to the lipid surface. ${ }^{17}$ The size of the liposomal vesicles significantly influences drug distribution. Large ( $>1 \mathrm{~m}$ ) MLV formulations are usually not used as antibiotic carriers, but SUVs of $\sim 100 \mathrm{~nm}$ exhibited high efficacy in the eradication of bacterial pathogens. ${ }^{18}$

The advantages of liposomal drug delivery: There are some antibiotics of limited application in healthcare because of toxicity or weak biodistribution and pharmacokinetics. Despite very efficient antibacterial activity, these drugs can only be used as last chance treatment when the risk of severe side effects is high. Encapsulation of the drugs in lipid vesicles is a good solution for designing the required pharmacokinetic and pharmacodynamic properties. ${ }^{19}$

a) There are many advantages of liposomes as antibiotic carriers:

b) Improved pharmacokinetics and biodistribution. c) Decreased toxicity.

d) Enhanced activity against intracellular pathogens.

e) Target selectivity.

f) Enhanced activity against extracellular pathogens, in particular to overcome bacterial drug resistance.

g) The variety of liposomal formulations allows the design of effective antibiotic forms and subsequent therapeutic success. ${ }^{20}$

Improved pharmacokinetics and biodistribution, decreased toxicity: There is much evidence of the benefits of liposomes as antibiotic delivery systems. The advantage of liposomal carriers is the possibility of a gradual and sustained release of antibiotics during drug circulation in the body. This allows maintaining the proper drug concentration for a relatively long term. In comparison, administration of the free antibiotic exhibits a quick and short effect and requires several doses per day. ${ }^{21}$ Drug encapsulation in liposomal vesicles improves the pharmacokinetics and also protects antibiotics against the hydrolytic activity of enzymes and chemical and immunological deactivation. Conventional liposomes applied by intravenous administration are recognized as foreign antigens by the immunological system and are opsonised. This activates nonspecific defence mechanisms and the liposomes are taken up by the mononuclear phagocyte system (MPS), which leads to lower blood circulation time and fast blood clearance. This phenomenon (phagocytosis of liposomes) is desirable for intracellular pathogen eradication, but unfavorable for other kinds of infection..$^{22,23}$ The MPS uptake rate depends on several liposomal properties, such as size, charge, and fluidity. The blood clearance of small vesicles $(\sim 100 \mathrm{~nm})$ rises to several hours, in comparison with several minutes for MLV formulations. Rigid and uncharged vesicles circulate longer than fluid and charged ones. It was demonstrated that increased capillary permeability at the infection site caused a high local concentration of liposomes. Additionally, the presence of bacterial antigens induced an inflammatory response, which led to a further increase in liposome extravagation. These mechanisms significantly improved the antibacterial activity of the encapsulated antibiotics and allowed the eradication of pathogen from the infected tissue..$^{24,25}$

Enhanced activity against intracellular pathogens and overcoming bacterial drug resistance: The application of liposomes as a drug delivery system was very successful in eradicating intracellular pathogens. Liposomes were applied to various types of infections. In the treatment of diseases caused by intracellular bacteria, rigid conventional liposomal vesicles and PEG-coated ones improved drug retention in the proper tissues, provided sustained release, decreased toxicity, and enhanced the concentration at the site of infection. Some experiments focused on tuberculosis, a severe and difficult to treat infection. It was shown that the application of liposomal forms of isoniazid, rifampin, and clarithromycin was very effective. ${ }^{27,28}$ There is also the possibility of controlled drug release by using $\mathrm{pH}$-sensitive liposomes. Their structure is stabile in the blood circulation, but in an altered $\mathrm{pH}$ environment (in phagolysosomes), an unstable membrane allows leakage of drug content. The $\mathrm{pH}$-sensitive PEG-coated vesicles composed of lipids were applied to intracellular pathogens such as Salmonella sp. and L. monocytogenes. ${ }^{28}$

Target selectivity: Intensive research on drug carriers demonstrated the possibility to target liposomes to particular tissues, organs, 
and even microorganisms. ${ }^{30}$ Target selectivity of liposomal drug formulations may be achieved by:

a. Addition of specific immunoglobulins; addition of proteins.

b. Addition of specific oligosaccharide chains.

c. Construction of $\mathrm{pH}$-sensitive vesicles.

d. Construction of thermo-sensitive vesicles, the composition of the vesicle surface conditions, the type o specific and nonspecific interaction with the target. ${ }^{31}$

In the case of nonspecific action, the charge of the membrane plays the main role. Eukaryotic and bacterial cells possess negatively charged surfaces, which is why positively charged liposomal vesicles exhibited the strongest vesicle-cell interactions. ${ }^{32}$ Specifically targeted liposomes are equipped with proteins, antibodies, or immunoglobulin fragments which have affinity to specific receptors located on the target surface (infected cells or pathogens). Specifically coated vesicles could be directed toward particular infected tissue or to strictly defined pathogens. Liposomes as drug carriers are very promising in preventing biofilm formation and treatment. The cationic liposomes were more efficient than anionic in adhering to skin bacteria. ${ }^{33}$ The researchers proposed that lectin-carbohydrate interactions are the principle mechanism for drug delivery to plaque-forming bacteria. The interactions between vesicles and epitomes expressed on the bacterial cell surface, such as glycocalyx, were studied and it was shown that polysaccharide-coated vesicles were an efficient system of metronidazol delivery to periodontal pocket biofilm and inhibition of pathogenic bacteria. The addition of zinc particles significantly increased the inhibitive effect on microbial growth and a synergic effect between the applied antimicrobials and zinc was noted. As mentioned previously, cationic formulations of liposomes exhibited significant adherence to the skin-associated bacteria S. epidermidis (Table 1). ${ }^{34}$

Table I Some of Liposomal antibiotics used for intracellular bacteria eradication ${ }^{29}$

\begin{tabular}{ll}
\hline Antibiotic & Bacteria \\
\hline Ampicillin & $\begin{array}{l}\text { Listeria monocytogenes Brucella melitensis; Brucella } \\
\text { abortus }\end{array}$ \\
Gentamicin & $\begin{array}{l}\text { Salmonella enterica serovar Typhimurium, Listeria } \\
\text { monocytogenes }\end{array}$ \\
Ciprofloxacin & $\begin{array}{l}\text { Salmonella enterica serovar Typhimurium, Francisella } \\
\text { tularensis }\end{array}$ \\
\hline
\end{tabular}

The disadvantages of liposomal antibiotics: One of the disadvantages of liposomal antibiotics is the short shelf-life of lipid vesicles, which limits drug stability. Short shelf lives can be conditioned by both physical and chemical processes. Chemical instability can be observed mainly due to the hydrolysis of ester bonds or the oxidation of unsaturated acyl chains of the lipids used to construct the liposomal vesicles. ${ }^{35}$ These processes occur in both synthetic and natural phospholipids. Moreover, besides hydrolysis and oxidation, per oxidation of unsaturated acyl chain bond is also possible (e.g. egg and soybean). Phospholipids from natural sources also exhibit structural diversity in their acyl chains, which causes differences in the stability and unique composition of liposomes. In this regard, the stability of liposomal drugs in vitro depends mainly on lipid composition, with storage temperature playing an important role. It is possible to prevent oxidation by adding antioxidant components or by freeze-drying. A low storage temperature also prevents hydrolysis. ${ }^{36}$ The physical instability of liposomal drugs leads to drug leakage from the lipid vesicles. The highest membrane permeability and fastest leakage of content can be observed at the lipid phase transition. It is higher in the liquid than in the gel phase. The problem with liposomal drug stability occurs mainly in cases of in vivo administration. Under physiological conditions, stability is usually low and depends on the interaction of the liposomal membranes with components of body fluids. ${ }^{37}$ Lipids that are transferred from the liposomal membrane to plasma lipoproteins in blood cause changes in liposome properties and release the drug. This process is most evident in liposomal formulations containing short-chain lipids or those with fluid membranes. Fluid liposomes release their content a few minutes after intravenous administration. The stability of liposomal vesicles can be enhanced by the addition of cholesterol, which stabilize their membrane and fluidity. Drug leakage increases in vivo in liposomes carrying a net charge. ${ }^{38}$ Another aspect of the physical instability of liposomes is the aggregation and fusion of liposomal vesicles. Both lead to changes in liposome size, which influences the in vivo therapeutic efficacy of the drug. A very important aspect of drug preparation is sterility. Sterilization procedures for liposomal antibiotics cannot involve the use of heat, irradiation, or chemical agents. Lipids are very sensitive to high temperatures and easily undergo oxidation and hydrolysis. ${ }^{39}$

\section{Nanomedicine}

Nanotechnology is the engineering and manufacturing of materials at the atomic and molecular scale. In its strictest definition from the National Nanotechnology Initiative, nanotechnology refers to structures roughly in the $1-100 \mathrm{~nm}$ size regime in at least one dimension. Despite this size restriction, nanotechnology commonly refers to structures that are up to several hundred nanometers in size and that are developed by top-down or bottom-up engineering of individual components. The application of nanotechnology to drug delivery is widely expected to change the landscape of pharmaceutical and biotechnology industries for the foreseeable future. ${ }^{39}$ The development of nanotechnology products may play an important role in adding a new armamentarium of therapeutics to the pipelines of pharmaceutical companies.

Using nanotechnology, it may be possible to achieve:

a. Improved delivery of poorly water-soluble drugs.

b. Targeted delivery of drugs in a cell- or tissue-specific manner.

c. Transcytosis of drugs across tight epithelial and endothelial barriers.

d. Delivery of large macromolecule drugs to intracellular sites of action.

e. Co-delivery of two or more drugs or therapeutic modality for combination therapy.

f. Visualization of sites of drug delivery by combining therapeutic agents with imaging modalities.

g. Real-time read on the in vivo efficacy of a therapeutic agent. ${ }^{40}$

Structure and properties of nanoparticles: The structure of nanoparticles allows better retention of the drug inside the polymeric network and then can be slowly degraded by esterase action. Monomers with long side chains are preferred since the acute toxicity of these 
polymers is greatly reduced. There are two types of nanoparticles: nanospheres (solid framework) and nanocapsules (liquid central cavity surrounded by a wall). Only nanospheres have been well studied for antibiotic delivery and will be described here. Since the conventional polymers are hydrophobic, highly polar compounds are less suitable for obtaining efficient loading. The classical nanoparticles are prepared by emulsion polymerization of alkylcyanoacrylic monomers in the presence of the drug. This reaction is induced by a nucleophilic agent. ${ }^{41}$ In some cases, the antimicrobial agent may itself induce the anionic polymerization of the monomer. The possible linkage of the drug to the polymer by a covalent bond could lead to its partial or complete non availability. Therefore, the antimicrobial activity of the nanoparticle drug formulation must be always compared in vitro with the free drug as control. The release rate of antibiotics from nanoparticles was found to be highly correlated with the degradation rate of the polymer by esterase. Thus, the release from nanoparticles is low in esterase-free medium but is greatly increased in the presence of carboxyesterase. Endocytosed by phagocyte cells, the colloidal carriers can be degraded in endosomes by lysosomal esterases. Usually, the entrapment efficiency is increased by using monomers with long side chain..$^{42}$ Nanoparticles have been fabricated using a variety of materials, including poly (lactide-co-glycolide) (PLGA); poly-lactic acid (PLA); polymethacrylic acid (PMA); polyethylene glycol (PEG);"natural" polymers such as chitosan, gelatin, or alginate; and other materials such as lipids, gold, and silica. ${ }^{43}$

\section{Advantages of using nonomediciene}

Biocompatibility and nano toxicity: Biocompatibility is an important feature of any drug delivery system, and the goal is to minimize nonspecific cytotoxic effects to healthy tissues while maximizing drug effects at the target tissue or against invasive pathogens. PLGA displays good biocompatibility, biodegradability, suitable degradation kinetics, and mechanical properties and is easy to process. For this reason, PLGA is an attractive candidate for nanoparticle-based drug delivery systems, and there is a large body of ongoing research in this area. ${ }^{44}$ Other polymers such as PLA, PMA, PEG, chitosan, gelatin, and alginate also show promise as drug delivery vehicles due to their biocompatible properties. PEG may be used as a "coating" to prevent the rapid removal of nanoparticles from the bloodstream by the mononuclear phagocytic system, which may increase nanoparticle circulation time and theoretically improve the therapeutic capacity of the nanoparticle. ${ }^{45}$

Cellular penetration and intracellular delivery: One critical challenge in treating intracellular pathogens is to get enough drugs to reach the pathogen within an intracellular compartment. Nanoparticles may reside within acidic endolysosome compartments following endocytosis, and premature drug release within this acidic compartment may cause drug degradation and render treatment ineffective. It is therefore important for the nanoparticle to escape this compartment and gain access to the cytosol where either the drug cargo may be directly released or the nanoparticle goes on to further target a specific organelle. For instance, PLGA nanoparticles carrying doxorubicin are reportedly capable of escaping the endolysosomal compartment by a reversal of their surface charge. This allows the particles to interact with the membrane and escape into the cytosol where the doxorubicin is released. ${ }^{46}$ There are a number of sources that report time- and concentration-dependent uptake of nanoparticles by a variety of cell types such as smooth muscle cells, endothelial cells, macrophages, and tumor cells. The uptake of PLGA nanoparticles containing bovine serum albumin as a model drug was found to be concentration dependent in human vascular endothelial cells, approaching first-order kinetics. An in vitro uptake and cellular trafficking study using mesoporous hybrid silica nanoparticles demonstrated that the particles were internalized by receptor-mediate endocytosis, were localized in the endocytic compartment, and then released their cargo within the cytosol. ${ }^{47}$

\section{Targeting to specific cell types}

The goal of targeting nanomedicines to specific cell populations is to increase the therapeutic efficacy of the drug while minimizing damage to healthy cells and tissues, thereby reducing the incidence and severity of side effects. Currently, the majority of experiments demonstrating the targeting of nanoparticles are related to cancer/ tumor targeting, although these same concepts can be applied to pathogen-infected cells and the intracellular compartments where the pathogens reside..$^{48}$ Nanoparticles can be targeted toward specific cell populations by conjugating targeting ligands to the surface of the nanoparticles. These targeting ligands can be attached directly to nanoparticle surfaces or via a spacer (eg, PEG), which acts to enhance the flexibility of the targeting ligand and increase the likelihood that it will bind the appropriate receptor on the target cell. There are a variety of methods available to attach ligands to nanoparticle surfaces. Targeting ligands can include antibodies (whole or fragment), receptors or receptor ligands, peptides, aptamers, or other small molecules. A few examples are presented here. ${ }^{49}$ When incorporating antibodies on nanoparticle surfaces, it is important to consider whether attachment will affect the binding site or structure of the antibody. Hybrid lipid nanoparticles composed of PLGA, phospholipids, and an outer PEG layer have shown targeting capacity to pancreatic cancer cells when coupled with an anti-carcinoembryonic antigen (CEA) half-antibody. ${ }^{50}$

Site-specific and tunable drug release: In order to effectively eradicate intracellular pathogens, drugs that are intended to kill the pathogens directly should reach the intracellular locations of infected host cells. The locations may include phagosomes, vacuoles, cytosol, and nucleus and may interact with the Golgi apparatus or endoplasmic reticulum. With proper engineering and design, nanoparticles can be tailored to carry their drug payloads into the infected cells and then release the drug within specific intracellular compartments. One way to accomplish site-specific drug release is to use $\mathrm{pH}$-responsive polymers. ${ }^{51}$ In one of the studies, short peptides were conjugated to $\mathrm{pH}$-responsive polymers designed specifically to disrupt the endosomal membrane at $\mathrm{pH} 5.5$ and subsequently release the peptide into the cytosol. The polymers had no membrane-disruptive activity at pH 7.4 due to a "masking" PEG group, which is later cleaved to expose the membrane-disruption domain at $\mathrm{pH}$ 5.5. Unconjugated peptide was located primarily in the lysosome, indicating that the peptide itself was unable to escape into the cytosol. This polymer technology may allow for local drug delivery to the cytosol, although it is more critical to reach the specific intracellular location of the pathogen, such as a vacuole or the nucleus..$^{52}$ It has been demonstrated that nanoparticles can be specifically targeted to mitochondria or nucleus and may be capable of entering vacuoles where pathogens such as Salmonella may reside during an infection process. Although intracellular pathogens do not typically live within mitochondria, 
pathogens such as Listeria monocytogenes can secrete toxins that interfere with normal mitochondrial function. So the ability to target mitochondria may provide a means to treat these types of infection and attenuate the effects of secreted toxins. ${ }^{53}$

\section{Disadvantage of using nanoparticles}

Of course, there are valid arguments against the use of nanomedicine, particularly around the issue of toxicity. As explained in the Scientific American article "Nano-risks: A Big Need for a Little Testing", Elements at these microscopic levels can exhibit different properties than they do normally. Furthermore, every nanoparticle is unique, and sometimes the effects or two of the same nanoparticles are not consistent. Thus, some nanoparticles might become dangerous for animals and humans. It has been shown that even nanoparticles that naturally occur in our body can have a serious effect on both our short term and long term health. If these naturally created nanoparticles can harm us, then it would not be wise to proceed with using ones that are artificially engineered with first considering the possible effects and consequences. If nanomedicine was expanded to nanorobotics, then we would need to consider the possible effects of a glitch in the programming, and how sever the effects must be. This reminds us that before nanomedicine can be used extensively, it will need to go through a rigorous process of testing to make sure it doesn't do more harm than good. ${ }^{54}$ Another disadvantage of nanotechnology is the enormous financial costs associated with it. As said in a report by the ETC group, Nanotech Rx, "the global health crisis doesn't stem from a lack of science innovation or medical technologies; the root problem is poverty and inequality. New medical technologies are irrelevant for poor people if they aren't accessible or affordable." The various natural polymers like gelatin, albumin and alginate are used to prepare the nanoparticles. However they have some inherent disadvantages like poor batch-to batch reproducibility, prone to degradation and potential antigenicity. ${ }^{55}$ Nanomedicine, like all technology, can also be used for malicious purposes. Much of the proposed technology and treatment that nanomedicine will bring can be used for purposes other than originally intended. This leads to problems of ethics and privacy. Nanorobots that could monitor the level of insulin in people in diabetes could also be misused by government and corporations trying to increase surveillance of citizens. Such technology can also be used for military purposes (Table 2 ). ${ }^{10}$

Table 2 Biocompatible nanoparticles discussed in this review ${ }^{54}$

\begin{tabular}{ll}
\hline Type of nanoparticle & Materials used \\
\hline & Poly lactide-co glycolide (PLGA) \\
Synthetic or man-mad polymerse & Poly-lactic acid (PLA) \\
& Polymethacrylic acid (PMA) \\
& Polyethylene glycol (PEG) \\
& Chitosan \\
& Gelatin \\
Natural polymers & Alginate \\
& Gold \\
Other types of nanoparticle & Lipids \\
\hline
\end{tabular}

\section{Clinical uses of liposomal and nanodelivery system}

Nanomedicine has been approved by the Food and Drug Administration for several biomedical applications, including surgical sutures, implants, and prosthetic devices. PLGA micro- or nanoparticles have also been used for a variety of drug delivery applications. Chitosan and alginate are polymers derived from natural sources and may offer more "friendly" conditions for the encapsulation or incorporation of DNA or peptides, because the use of organic solvents can be avoided. ${ }^{56}$ Liposomes are composed of lipid bilayers surrounding a hydrophilic "core" and can be designed to incorporate hydrophobic drugs within the lipid bilayer or hydrophilic drugs within the aqueous core. Liposome-encapsulated aminoglycosides (such as gentamicin) have shown higher therapeutic efficacy than conventional amino glycoside preparations in the liver and spleen using a murine $S$. typhi infection model. Amphotericin B (an antifungal) has also been encapsulated in lipids and administered to mice with an Aspergillus fumigatus infection. Liposome-treated mice have survived longer than mice treated with other amphotericin-B preparations, with reduced renal toxicity and a prolonged drug circulation time. These liposome formulations could be a better treatment for diseases affecting the liver or spleen (eg, typhoid fever, hepatitis). ${ }^{57}$ Lipid nanocapsules (LNCs) are another type of lipidmediated delivery system under exploration. LNCs are considered a "hybrid" between a polymer nanoparticle and a liposome, with an oily core surrounded by a membrane composed of PEGylated surfactants. LNCs have shown promise in vitro and some animal models encapsulating anticancer drugs such as paclitaxel, doxorubicin, and Etoposide.

The results of these studies have indicated higher intracellular drug delivery and reduced tumor size in vivo when LNC formulations were administered. ${ }^{57}$ In contrast to these "soft" polymer-based nanoparticle systems, metals such as gold have also been explored as potential drug delivery vectors. Gold is an attractive drug delivery vector due to the ease with which biomolecules such as protein or DNA can be attached to the gold surface using thiol chemistry. This process can also allow attachment of multiple targeting or functional groups to the nanoparticle surface to produce a multifunctional nanoparticle. Although gold nanoparticles can be easily functionalized, these nanoparticles may accumulate in tissues over time because they are not biodegradable. ${ }^{57}$ The effects of long-term nanoparticle accumulation are unknown, so in many cases it may be better to use a material that is fully biodegradable. Silica-based compounds are another option, as the biodegradation of silica avoids tissue accumulation concerns, and it has been demonstrated that a variety of agents have been successfully incorporated into silica-based nanoparticles for drug delivery applications. Although each of these materials offers its own set of characteristics and biocompatible properties, some materials may be more suited to certain applications than others. It is important to 3 determine the desired properties of the nanomedicine for defined applications. $^{58}$

\section{The major difference between liposomes and nanoparticles}

Liposomes are "phospholipid bubbles with a membrane bilayer structure" which, because of their size, are located on the border 
between micro- and nanotechnology. Liposomes were among the first nanotechnologies to be used in clinical trials and among the first to appear on the market. ${ }^{59}$ There is much less known about the development of nanoparticles conveying the greater interest in liposomes with their membrane-like composition. However, the stability of liposomes is often contested and other types of carriers are studied in order to have better control over the delivery of drugs. Owing to their polymeric nature, nanoparticles may be more stable than liposomes in biological fluids and during storage. ${ }^{60}$ In general liposomes and nanoparticles are differ based on their structural constituents and their size. The major constituent of liposome is phospholipid; but, in nanoparticle, polymers of different compounds are used instead of lipid.

\section{Conclusion and recommendations}

The discovery of liposomes as universal carriers allowed the development of various delivery systems for enzymes, DNA, drugs, and other chemical compounds. The possibility of modifying the vesicles formulation plays a major role in their wide application. The manipulation of different physicochemical properties of liposomes, such as size, lipid type, the lipid composition of the liposomal formulation, the charge on the liposomal surface, and fluidity of the liposomal membrane, enables the design of particular carriers with the desired pharmacokinetic and pharmacodynamic properties. With recent scientific advances, it will be increasingly feasible to engineer targeted or multifunctional nanotechnology products for therapeutic applications. With the correct combination of an optimally engineered vehicle, a suitable drug, and a "killer app" disease, the benefit of a targeted drug delivery system over the equivalent non-targeted system is expected to be substantial. In the near- and medium-term, we can expect the emergence of many nanotechnology platforms for drug delivery applications. While both organic and inorganic technologies are under development, controlled-release polymer technologies and liposomes will likely continue to have the greatest clinical impact for the foreseeable future. These are exciting times for nanotechnology research, and the pace of scientific discovery in this area is gaining momentum. It is widely accepted that with continued resources, medicine and the field of drug delivery will be an important beneficiary of nanotechnology for years to come.

Based on the above conclusion the following recommendations are forwarded:

A. It is obvious that not only clinical trials, but also nanomedicine preparation is extremely time and cost consuming, but it should not limit the wide application of these drug preparations in the health service; considering the welfare of patients.

B. Using Nanomedicine is important to avoid or prevent further development of pathogen resistance, which is becoming a major problem for the management of disease.

C. It should be avoided using nanotechnology for malicious purposes or for any action other than originally intended.

\section{Acknowledgements}

Praise to God the most powerful, self-confidence and creator of all things for his mercy up us.

\section{Conflict of interest}

The author declares that there is no conflict of interest.

\section{References}

1. Vyas SP, Khar RK. Targeted and controlled drug delivery: Novel Carrier Systems. Int J Pharmaceut. 2003;267(Suppl 1-2):157.

2. Kumari A, Yadav SK, Yadav SC. Biodegradable polymers nanoparticles based drug delivery systems. Colloids Surf B Biointerfaces. 2010;75(1):118.

3. Abeylath SC, Turos E. Drug delivery approaches to overcome bacterial resistance to beta-lactam antibiotics. Expert Opin Drug Deliv. 2008;5(9):5931-5949

4. Bakker Woudenberg IA. Long-circulating sterically stabilized liposomes as carriers of agents for treatment of infection or for imaging infectious foci. Int J Antimicrob Agents. 2002;19(4):299-311.

5. Allen TM. Liposomal drug formulations. Rationale for development and what we can expect for the future. Drugs. 1998;56(5):747-756.

6. Allen TM, Martin FJ. Advantages of liposomal delivery systems for anthracyclines. Semin Oncol. 2004;31(6 Suppl 13):5-15.

7. Bangham AD. Surrogate cells or Trojan horses. The discovery of liposomes. Bioessays. 1995;17(12):1081-1088.

8. Bakker-Woudenberg IA, Storm G, Woodle MC. Liposomes in the treatment of infections. J Drug Target. 1994b;2(5):363-371.

9. Andrea LA, Li B. Review: Nano medicine as an emerging approach against intracellular pathogen. Int J Nanomedicine. 2011;6:3281-3293.

10. Bakker-Woudenberg IA, Storm G, Woodle MC. Liposomes in the treatment of infections. J Drug Target. 1994b;2(5):363-371.

11. Bakker-Woudenberg IA, Lokerse AF, ten Kate MT, et al. Liposomes with prolonged blood circulation and selective localization in Klebsiella pneumoniae-infected lung tissue. J Infect Dis. 1993;168(1):164-171.

12. Catuogno C, Jones MN. The antibacterial properties of solid supported liposomes on Streptococcus oralis biofilms. Int J Pharm. 2003;257(12):125-140

13. Chapman D. Physicochemical properties of phospholipids and lipid-water systems. In: Gregoriadis G, editor. Liposome technology. Boca Raton: CRC Press; 1984. 1-18 p.

14. Chiang CY, Schaaf HS. Management of drug-resistant tuberculosis. Int J Tuberc Lung Dis. 2010;14:672-682.

15. Cordeiro C, Wiseman DJ, Lutwyche P, et al. Antibacterial efficacy of gentamicin encapsulated in $\mathrm{pH}$-sensitive liposomes against an in vivo Salmonella enterica serovar Typhimurium intracellular infection model. Antimicrob Agents Chemother. 2000;44(3):533-539.

16. Deol P, Khuller GK. Lung specific stealth liposomes: stability, biodistribution and toxicity of liposomal antitubercular drugs in mice. Biochim Biophys Acta. 1997;1334(2-3):161-172.

17. Dijkstra J, van Galen WJM, Regts J, et al. Uptake and processing of liposomal phospholipids by Kuppfer cells in vitro. Eur J Biochem. 1985;148(2):391-397.

18. Drulis Kawa Z, Gubernator J, Dorotkiewicz JA, et al. In vitro antimicrobial activity of liposomal meropenem against Pseudomonas aeruginosa strains. Int J Pharm. 2006;315(1-2):59-66.

19. Drulis Kawa Z, Dorotkiewicz JA. Liposomes as delivery systems for antibiotics. Int J Pharm. 2010;387(1-2):187-198.

20. Ferrari M. Cancer Nanotechnology: Opportunities and Challenges. Nat Rev Cancer. 2005;5(3):161-171.

21. Gregoriadis G. The carrier potential of liposomes in biology and medicine. N Engl J Med. 1976;295(14):765-770. 
22. Gregoradis G. Liposome preparation and related techniques. In: Gregoriadis G, editor. Liposome Technology. Boca Raton: CRC Press; 1993. 1-63 p.

23. Gregoriadis G. Engineering liposomes for drug delivery: progress and problems. Trends Biotechnol. 1995;13(12):527-537.

24. Hamidi M, Azadi A, Rafiei P. Pharmacokinetic consequences of pegylation. Drug Deliv. 2006;13(6):399-409.

25. Medina C, Rahme K, Arcy DM, et al. Poloxamer mixed micelles for delivery of gambogic. Int J Nanomedicine. 1996;10:407-409.

26. Hu CM, Kaushal S, Cao HST, et al. Half-antibody functionalized lipid-polymer hybrid nanoparticles for targeted drug delivery to carcinoembryonic antigen presenting pancreatic cancer cells. Mol Pharm 2010;7(3):914-920

27. Huynh NT, Passirani C, Saulnier P, et al. Lipid nanocapsules: a new platform for nanomedicines. Int J Pharm. 2009;379(2):201-209.

28. Joralemon MJ, McRae S, Emrick T. PEGylated polymers for medicine: from conjugation to self-assembled systems. Chem Comm. 2010;46(9):1377-1393

29. Jung SK, Lim DH, Jung SH, et al. Amphotercin B-entrapping lipid nanoparticles and their in vitro and in vivo characteristics. Eur J Pharm Sci. 2009;37(3-4):313-320.

30. Gilbert P, Collier PJ, Brown MRW. Influence of growth rate on susceptibility to antimicrobial agents: biofilms, cell cycle, dormancy, and stringent response. Antimicrob Agents Chemother. 1990;34(10):1865-1868.

31. Kohane DS, Tse JY, Yeo Y, et al. Biodegradable polymeric microspheres and nanospheres for drug delivery in the peritoneum. J Biomed Mater Res. 2006;77(2):351-361.

32. Lasic DD. Novel applications of liposomes. Trends Biotechnol, 1998;16(7):307-321.

33. Krieger J, Childs S, Klimberg I. UTI treatment using liposomal amika in Berlin. Clinical Microbiology. 1999;5(Suppl 3):136-144.

34. Martel S. Disadvantage of nanomedicine. Int J Nanomedicine. 2009;50:15 .

35. Mouritsen OG, Jorgensen K. A new look at lipid-membrane structure in relation to drug research. Pharm Res. 1998;15(10):1507-1519.

36. Murthy N, Campbell J, Fausto N, et al. Bio- inspired pH-responsive polymers for the intracellular delivery of biomolecular drugs. Bioconjug Chem. 2003;14(2):412-419.

37. Panyam J, Zhou WZ, Prabha S, et al. Rapid endolysosomal escape of nanoparticles. Implications for drug and gene delivery. FASEB $J$ 2002;16(10):1217-1226.

38. Pinto Alphandary H, Andremont A, Couvreur P. Targeted delivery of antibiotics using liposomes and nanoparticles: research and applications. Int $J$ Antimicrob Agents. 2000;13(3):155-168.

39. Reis CP, Neufeld RJ, Vilela S, et al. Review and current status of emulsions/dispersions technology using an internal gelation process for the design of alginate particles. J Microencapsul. 2006;23(3):245-257.

40. Rosenholm JM, Peuhu E, Eriksson JE, et al. Targeted intracellular delivery of hydrophobic agents using mesoporous hybrid silica nanoparticles as carrier systems. Nano Lett. 2002;9(9):3308-3311.

41. Sapra P, Allen TM. Ligand-targeted liposomal anticancer drugs. Prog Lipid Res. 2003;42(5):439-462.
42. Scheinberg DA, Villa CH, Escorcia FE, et al. Conscripts of the infinite armada: systemic cancer therapy using nanomaterials. Nat Rev Clin Oncol. 2010;7(5):266-276

43. Schiffelers R, Storm G, Bakker-Woudenberg I. Liposome encapsulated aminoglycosides in pre-clinical and clinical studies. J Antimicrob Chemother. 2001;48(3):333-344

44. Schiffelers RM, Storm G, Bakker-Woudenberg IA. Host factors influencing the preferential localization of sterically stabilized liposomes in Klebsiella pneumoniae-infected rat lung tissue. Pharm Res.2001;18(6):780787.

45. Seleem MN, Munusamy P, Ranjan A, et al. Silica-antibiotic hybrid nanoparticles for targeting intracellular pathogens. Antimicrob Agents Chemother. 2009;53(10):4270-4274.

46. Sessa G, Weissmann G. Phospholipid spherules (liposomes) as a mode for biological membranes. J Lipid Res. 1968;9(3)310-318.

47. Sihorkar V, Vyas SP. Biofilm consortia on biomedical and biological surfaces: delivery and targeting strategies. Pharm Res. 2001;18(9):1247-1254.

48. Sharma A, Sharma US. Liposomes in drug delivery: progress and limitations. Int J Pharm. 1997;154(2):123-140.

49. Stavru F, Bouillaud F, Sartori A, et al. Listeria monocytogenes transiently alters mitochondrial dynamics during infection. Proc Natl Acad Sci USA. 2011;108(9):3612-3617.

50. Heuer D, Lipinski AR, Machuy N, et al. Chlamydia causes fragementation of the golgi compartment to ensure reproduction. Nature. 2009;457(7230):731-735.

51. Swenson CE, Popescu MC, Ginsberg RS. Preparation and use of liposomes in the treatment of microbial infections. Crit Rev Microbiol. 1988;15(Suppl 1):S1-S31

52. Torchilin VP. Recent advances with liposomes as pharmaceutical carriers. Nat Rev Drug Discov. 2005;4(2):145-160

53. Ulrich AS. Biophysical aspects of using liposomes as delivery vehicles. Biosci Rep. 2002;22(2):129-150.

54. Voinea M, Simionescu M. Designing of 'intelligent' liposomes for efficient delivery of drugs. J Cell Mol Med. 2002;6(4):465-474.

55. Watve RM, Bellare JR. Manufacture of liposomes: a review. Current Sci. 1995;68(7):715-724.

56. Webb MS, Boman NL, Wiseman DJ, et al. Antibacterial efficacy against an in vivo Salmonella typhimurium infection model and pharmacokinetics of a liposomal ciprofloxacin formulation. Antimicrob Agents Chemother. 1998;42(1):45-52.

57. Wang M, Thanou M. Targeting nanoparticles to cancer. Pharmacol Res 2010;62(2):90-99.

58. Young. Review: Disadvantage of Nanomedicine. J Nuclear Med. 2009;50:1-4

59. Zhang L, Gu FX, Chan JM, et al. Nanoparticles in Medicine: Therapeutic Applications and Developments. Clin Pharmacol Ther. 2008;83(5):761769

60. Zuidam NJ, Lee SS, Crommelin DJ. Sterilization of liposomes by heat treatment. Pharm Res. 1993;10(11):1591-1596. 